peroxidation index $-\mathrm{MDA}$ increased significantly after tachypacing in ATP group (from $1.99 \pm 0.51$ to $2.94 \pm 0.78 \mathrm{nmol} / \mathrm{ml}, \mathrm{p}<0.05$ ), but no change in $\mathrm{A}+\mathrm{O}$ group. 2. Compare to the result at the same time in $\mathrm{R}+\mathrm{O}$ group, the ERP shortened dramatically $(\mathrm{p}<0.05)$ after tachypacing in ATP group; The Rate adaptive of ERP appeared nonperforming significantly after pacing in ATP group; The level of MDA increased $(p<0.05)$ after tachypacing in ATP group.

Conclusion Omeprazole could effectively suppressed tachypacinginduced electrical remodelling in rabbit AF model and greatly attenuated the oxidative stress by downregulating lipid peroxidation.

\section{e0008 EFFECT OF HIF-1A ON MSC TRANSPLANTATION THERAPY OF RAT ACUTE MYOCARDIAL INFARCTION}

doi:10.1136/hrt.2010.208967.8

${ }^{1}$ Li Xueyuan, ${ }^{2}$ Zhang Yang, ${ }^{1}$ Qi Guoxian. ${ }^{1}$ The First Hospital of China Medical University; ${ }^{2}$ The Centrol Affiliated Hospital of Shenyang Medical College

Aims To investigate the effect and mechanism of HIF-1a on rat AMI therapy by MSC transplantation. Materials and methods Rat acute myocardial infarction model is made through coronary anterior descending artery ligation. Rats are randomly divided into four groups which are sham operation group, pure infarction group, infarction \& MSC transplantation group and infarction \& HIF-1a transfected MSC transplantation group. Eight rats from every group are observed. The cell transplantation is carried out immediately after the acute myocardial infarction model is successfully made. The rats are put to death 4 weeks after the operation and the heart is isolated for weight measuring, heart chamber and myocardium thickness testing. We also observe the myocardial angiogenesis in and around the infarct myocardium through $\mathrm{HE}$ staining, and the distribution of transplanted cells in the myocardium tissue under immunofluorescence staining. Western blot and RT-PCR is used to test the expression of HIF-1a and VEGF in the myocardium.

Results About $37 \%$ of the operations on AMI model making are successful. More MSCs transfected with HIF-1 are alive after transplantation than other groups $(p<0.05)$. Heart weight and left ventricular chamber of the rats transplanted with MSCs transfected with HIF-1a are lower and smaller than the other three groups $(p<0.05)$, the thickness of the left ventricular well is much thicker than the others $(p<0.05)$. Capillary regeneration in and around the infarction area is greater than the others $(p<0.05)$. Higher expression of HIF-1a $(p<0.05)$ and VEGF $(p<0.05)$ can be detected in the myocardium of the arts transplanted with MSCs transfected with HIF-1a.

Conclusions HIF-1a could raise the survival rate of MSC in the infarct myocardium area. MSC transfected with HIF-1a could restrain myocardium remodelling after infarction, and raise the density of capillary around infarction, which might be the mechanism of the former.

\section{e0009 MULTIMODALITY MOLECULAR IMAGING OF ADIPOSE- DERIVED MESENCHYMAL STEM CELLS WITH VEGF IN HINDLIMB ISCHAEMIA MICE}

\section{doi:10.1136/hrt.2010.208967.9}

${ }^{1}$ Fan Weiwei, ${ }^{1}$ Cao Feng, ${ }^{1}$ Wang Yabin, ${ }^{2}$ Liu Junting, 'Li Shuang, 'Zhang Rongqing, ${ }^{1}$ Li Congye, ${ }^{1}$ Li Chengxiang, ${ }^{2}$ Tian Jie. ${ }^{1}$ Xijing Hospital; ${ }^{2}$ Xidian University

Background Peripheral arterial disease (PAD) is highly prevalent and particularly in elders, smokers, diabetics or patients with systemic atherosclerosis. Apart from the surgery and medication, stem cell transplantation offers promising approaches for therapeutic angiogenesis and tissue repair. In this study we try to use in vivo multimodality molecular imaging strategies to investigate adipose tissue-derived mesenchymal stem cells (MSCs) survival, function and relative mechanism.

Method MSCs were cultured from murine adipose tissue from transgenic mice, which carried double reporter genes: firefly luciferase (Fluc) and enhanced green fluorescent protein (eGFP), by collagenase digestion method. Hindlimb ischaemia animal model was created in male nude mice by ligating the proximal and distal femoral artery. MSCs $\left(1 \times 10^{5}\right)$ along with/without VEGF $(0.4 \mathrm{ng})$ were transplanted into ischaemic hindlimb. The animals were subjected to be imaged by bioluminescence imaging and CT scan. Laser Doppler perfusion imaging (LDPI) were used to show the spatiotemporal images of peripheral tissue blood perfusion. Micro-CT, histological and molecular analysis were tested to confirm the cells' location and angiogenesis anatomically and mechanically. Result The colour-coded index of LDPI was significantly higher in the MSCs-transplanted group than that in the control group from day 3 to 28 post cell transplantation. On day 3 after transplantation, the bioluminescence signals in MSCs with VEGF group were $4.6 \times 10^{6} \pm 2.5 \times 10^{5}$ photons/ $\mathrm{s} / \mathrm{cm}^{2} / \mathrm{sr}$, while in MSCs group were $2.8 \times 10^{6} \pm 3.1 \times 10^{5}$ photons $/ \mathrm{s} /$ $\mathrm{cm}^{2} / \mathrm{sr}$, respectively $(\mathrm{p}<0.01$ vs control). The signals of bioluminescence increased gradually from POD 3 to day 21, which proved survival and proliferation of the MSCs in the host. The group treated with MSCs and VEGF showed higher signals than that injected by MSCs only, which indicated the reinforcement of VEGF. Micro-CT angiography demonstrated more angiogenesis in the hindlimbs of the treated mice on day 21, which were also confirmed by molecular analysis. Histological analysis showed that MSCs therapy recovered vessel density compared with the control group.

Conclusion Bioluminescence fusion with CT scan provides higher detailed 3D imaging for monitoring MSCs in vivo. Angiogenesis activator VEGF might promote MSCs' beneficial function for hindlimb ischaemia therapy.

\section{e0010 ATORVASTATIN INHIBITS OXIDISED LOW DENSITY LIPOPROTEIN INDUCED DIFFERENTIATION OF RAW2647 MURINE MACROPAHGES INTO DENDRITIC LIKE CELLS}

doi:10.1136/hrt.2010.208967.10

Liu Hua Hu. Renji Hospital Medical College of Shanghai Jiaotong University

Dendritic cells (DCs) are professional antigen-presenting cells and have an important role in the pathogenesis of atherosclerosis. It has been confirmed that the optimal oxLDL dose $(10 \mathrm{ug} / \mathrm{ml})$ can induce approximatelly $74 \%$ RAW264.7 cells differentiate into dendritic-like cells in our previous work. In this study, we examined whether atorvastatin could inhibit the differentiation of mature macrophages into DCs induced by oxLDL, since statins are lipid-lowering drugs. After $24 \mathrm{~h}$ treatment with atorvastatin $(20 \mathrm{umol} / \mathrm{ml})$, almost all the RAW264.7 cells induced by oxLDL simultaneously remained in cell size and macrophages morphology compared with those induced by oxLDL alone. Flow cytometric analysis detected reduced dendritic cell surface markers (CD40, CD86, MHC Class II and CD1d, table 1). Moreover, atorvastatin-treated RAW264.7 cells induced by oxLDL shown functional changes including increased phagocytic ability (table 2) in a time-dependent manner and reduced TNF-a as well as IL-12 p70 (table 3) productin. On the whole, these data suggest dendritic-like cells origined from macrophages induced by oxLDL treatment can be inhibited by atorvastatin and this may contribute to the effect of statins on preventing the formation of atherosclerotic plaques.

Table 1. dendritic cell surface markers expression (\%) in $24 \mathrm{~h}$ RAW OxLDL OxLDL+atorvastatin CD40 6.09\% $\pm 0.48 \% 51.68 \% \pm 1.33 \%$ * 40.09\% $\pm 1.59 \% *$ CD86 5.00\% $\pm 0.62 \% 55.04 \% \pm 1.27 \% * 24.29 \% \pm$ $1.30 \% *$ CD1d MHC Class II 3.64\% $\pm 0.41 \% 5.09 \% \pm 0.13 \% 33.79 \% \pm$ $2.47 \% * 17.10 \% \pm 1.42 \% * 33.40 \% \pm 11.54 \% * 22.87 \% \pm 6.665 *$ 